Horizons philosophiques

\title{
De la nécessité de la vulgarisation en philosophie
}

\section{Lucien Bérubé}

Volume 8, numéro 2, printemps 1998

Défense et illustration de la vulgarisation philosophique

URI : https://id.erudit.org/iderudit/801074ar

DOI : https://doi.org/10.7202/801074ar

Aller au sommaire du numéro

\section{Éditeur(s)}

Collège Édouard-Montpetit

\section{ISSN}

1181-9227 (imprimé)

1920-2954 (numérique)

Découvrir la revue

\section{Citer cet article}

Bérubé, L. (1998). De la nécessité de la vulgarisation en philosophie. Horizons philosophiques, 8(2), 47-61. https://doi.org/10.7202/801074ar d'utilisation que vous pouvez consulter en ligne.

https://apropos.erudit.org/fr/usagers/politique-dutilisation/ 


\section{DE LA NÉCESSITÉ DE LA VULGARISATION EN PHILOSOPHIE}

Attardons-nous un moment à l'origine du mot philosophie, imitant en cela une règle de méthode que donne Aristote au commencement de son traité de La Politique1. À qui attribuaiton le nom de philosophe, au début? Cicéron, dans les Tusculanes ${ }^{2}$, nous apprend que le nom fut inventé par Pythagore pour désigner un type d'homme qui existait bien avant lui et qu'on avait l'habitude de dénommer par le vocable de sage. Et il précise : “C'est par l'étude des choses divines et humaines, puis celle des principes et des causes de toute chose, que, dans l'antiquité, on obtenait le beau nom de sage ${ }^{3}$. Les choses humaines désignant tout ce que l'homme produit par ses diverses activités et les choses divines désignant tout ce qui existe et qui n'est pas le résultat d'une activité de l'homme, donc désignant les choses produites par la nature (il ne s'agit évidemment pas ici des choses divines au sens où l'entend la théologie), Cicéron peut donc affirmer avec raison que l'étude du sage porte sur les principes et les causes de "toute chose». Ce sont les quelques individus qui s'adonnaient à cette sorte d'étude que les gens appelaient sages. Ayant pris conscience qu'en raison de son extrême difficulté une telle étude demeurait toujours inachevée, et que, conséquemment, personne ne pouvait jamais être dit proprement sage, Pythagore, peut-on supposer, crut bon de forger ce mot "philosophe», ami de la sagesse, c'est-à-dire chercheur de cette connaissance portant sur les principes et les causes de toute chose.

1. Aristote, La Politique, Livre 1, c. 2, 1252a 24-25, traduction J. Tricot, Paris : Librairie philosophique J. Vrin, 1962, Tome 1, p. 24 : "Dans ces conditions, si on considérait les choses à partir de leur origine, dans leur développement naturel, comme on le fait dans les autres domaines..."

2. Cicéron, Tusculanes, Livre V, section III, traduction Jules Humbert, Paris : Société d'Édition "Les Belles Lettres", 1960, Tome III, p. 109-111.

3. Ibid., no. 7, p. 109. 
Un autre point ressort de ce texte de Cicéron : «Pythagore, d'après une tradition recueillie par un auditeur de Platon, Héraclide du Pont, qui fut un savant distingué, s'était rendu à Phlionte et avait traité devant Léon, prince de Phlionte, certaines questions où il s'était montré savant et disert (docte et copiose disseruisse quaedam). Émerveillé de son intelligence et de son éloquence, Léon lui aurait demandé quelle était la science dont il se réclamait spécialement (quaesivisse ex eo qua maxime arte confideret), et voilà que Pythagore aurait répondu qu'en fait de spécialité il n'en avait aucune, mais qu'il était philosophe (artem quidem se scire nullam, sed esse philosophum) ${ }^{4}$ ". L'idée de cette citation est la suivante : ce qui rend Pythagore capable de discourir savamment (docte) et avec une grande richesse d'idées (copiose), ce n'est pas la connaissance d'une science particulière (ars, au sens de science ici), mais la philosophie, c'est-à-dire cette connaissance obtenue par l'étude des principes et des causes de toute chose.

En clair, cette page de Cicéron désigne l'intention de Pythagore en forgeant ce mot : signifier un type d'études ou de recherches, non pas celles portant sur des applications de théories, sur des conclusions particulières comme on en trouve en sciences, ou sur des techniques particulières et spécialisées, mais cette sorte de recherches qui concernent les principes. Cette même page de Cicéron identifie aussi une caractéristique des connaissances obtenues par cette sorte de recherches : la capacité de discourir et de juger d'une foule de choses parce qu'on les a comprises dans leurs principes, ce que ne permet pas, semble-t-il, une science particulière. Voilà deux points que nous apprend une brève étude de l'origine du mot philosophe ou philosophie.

II ne faudrait pas s'imaginer que ces deux caractéristiques, perçues à l'origine, c'est-à-dire au moment de la formation de ce mot, se sont évanouies au cours des âges. Bien au contraire! Elles sont encore expressément énoncées, entre autres, dans une page trop méconnue de Péguy, qu'il vaut la peine de citer :

4. Ibid., no. 8, p. 110. 
Dans toutes les occupations humaines la division du travail se fait normalement ainsi : se situant également et ensemble au lieu des postulats, principes, définitions, conditions et limites, et des situations faites, le savant et l'artiste, s'accordant très libéralement tout cela, qui est demandé, considérant tout cela comme allant de soi, comme vu et entendu, partant de ce point précis redescendent incontinent le cours de leurs sciences respectives et de leurs arts. Mais se situant également et ensemble avec eux à ce même point précis, à ce point de difficulté, le philosophe s'y assied, et il n'en veut plus démarrer avant que d'avoir éclairci ces difficultés, qui sont généralement inéclaircissables. De là vient sa dignité, son prix, singulier, sa grandeur et sa lamentable misère. De là vient que les autres le méprisent et le redoutent, et quelquefois le haïssent, haussent les épaules, mais quelquefois baissent les yeux...

Les autres descendent le fil de l'eau. II ne quittera plus ce poste que pour essayer, partant de ce point, de remonter plus haut encore, tournant délibérément le dos aux autres qui descendent le courant, de remonter plus haut encore dans des régions encore plus inaccessibles. Les autres suivent le fil de l'eau de l'art, et de la science, et de la vie. Lui au contraire, il a entrepris de remonter le courant de l'être. S'il peut5.

Cette page mérite d'être connue et d'être utilisée pour deux raisons. Pour une raison pédagogique d'abord : la comparaison qu'elle contient implicitement et qui peut être développée (les savants et les artistes semblables à un nageur qui descend «le fil de l'eau", et les philosophes à quelqu'un qui nage à contrecourant, tous les trois ayant un même point de départ), permet de manifester simplement le travail du philosophe et ses rapports avec les autres disciplines. Puis une deuxième raison, "scientifique" celle-là : cette page manifeste quelque chose d'essentiel à la philosophie, à savoir les deux caractéristiques que nous avons identifiées plus haut. En effet, que le

5. Charles Péguy, De la situation faite à l'histoire et à la sociologie, In Oeuvres en prose, 1898-1908, Bibliothèque de la Pléiade, Paris : Gallimard, 1959, p. 1005, 1006. 
philosophe se consacre à l'étude des principes, afin de les comprendre et de les justifier, afin de les éclaircir pour pouvoir par la suite les éclairer, ressort clairement de ce texte de Péguy. Que le philosophe se consacre à l'étude de notions intimement liées aux recherches des savants et des artistes, est aussi nettement affirmé : le travail des philosophes, des savants et des artistes prend son origine au même point de départ - les principes - : les philosophes essaient, «partant de ce point, de remonter plus haut encore", donc de comprendre et de justifier les principes, alors que les savants et les artistes cherchent, eux, des applications nouvelles de ces mêmes principes, à l'intérieur de leurs sciences et leurs arts respectifs, principes qu'ils considèrent "comme allant de soi, comme vu(s) et entendu(s)". C'est en raison de cette étude sur les principes dont se servent finalement les savants et les artistes (et on pourrait ajouter aussi les principes dont se servent tous les hommes dans leur vie) que les philosophes acquièrent la capacité de discourir sur une foule de choses, savamment (docte) et avec une grande richesse d'idées (copiose), comme le disait Cicéron.

II faut ajouter que l'histoire de la philosophie, dans son ensemble, témoigne de l'exactitude de ces deux caractéristiques de la philosophie, tout comme elle confirme aussi une autre caractéristique de la philosophie qui ressort de ces deux textes : sa difficulté. Je l'ai déjà indiqué, on peut supposer que c'est par humilité que Pythagore a inventé le mot philosophe, s'étant rendu compte de la difficulté à acquérir cette sagesse grâce à laquelle quelqu'un est dit sage. Et Péguy se réfère, lui, directement à cette difficulté lorsqu'il parle du travail du philosophe qui veut éclaircir les difficultés concernant les principes "qui sont généralement inéclaircissables», qui veut "remonter plus haut encore dans des régions encore plus inaccessibles", qui "a entrepris de remonter le courant de l'être».

Comme on le constate, tout ce que ces deux textes nous apprennent sur la philosophie est fondé sur la notion de principe. Qu'ont donc les principes pour rendre quelqu'un capable de juger, d'une certaine façon, de tout et pour être si diffi- 
ciles à saisir? Au tout début du livre D de La Métaphysique 6 , Aristote distingue divers sens du mot principe.

Ce mot désigne d'abord, selon lui, le point de départ d'un mouvement : si je vais de Québec à Montréal, le point de départ de mon déplacement, ou le principe de mon déplacement, est évidemment Québec. Manifestement cette signification correspond à la question : d'où est-ce que je pars? et désigne un point spatial. Mais comme le mouvement ne débute pas toujours à partir d'un point spatial, Aristote distingue un deuxième sens : «Le principe est aussi le meilleur point de départ pour chaque chose 7 ", signifiant par là que le point de départ correspond à la situation dans laquelle se trouve l'être qui effectue le mouvement. Ainsi dans l'exemple du trajet Québec-Montréal utilisé plus haut, Québec est bien, en soi, le point de départ; mais quelqu'un qui, parti de Québec pour Montréal, séjourne quelques jours à Trois-Rivières, repartira de cette ville pour terminer son trajet, cette ville correspondant à la situation dans laquelle se trouve l'individu. L'exemple que donne Aristote est cependant beaucoup plus éclairant - et surtout il est tout à fait adapté au propos de ce texte : “Par exemple, même dans la science, il ne faut pas parfois commencer par le commencement et par la notion première de l'objet, mais par ce qui peut le mieux en faciliter l'étude8". Illustrons cet exemple par le cas suivant : la théorie de la relativité peut être considérée comme un système en soi, ayant ses propositions de départ, ses démonstrations et ses conclusions. Dans ce cas, les principes de cette théorie sont ses propositions de départ, mais celles-ci ne peuvent pas être des principes pour un élève de Secondaire $\mathrm{V}$. On voit bien que ce deuxième sens du mot principe ne tient pas exclusivement compte du mouvement considéré en lui-même (ce que fait le premier sens), mais du sujet qui effectue ce mouvement. Les deux autres sens du mot principe, toujours selon Aristote, concernent la génération ou le devenir

\footnotetext{
6. Aristote, La Métaphysique, Livre D, c. 1, traduction J. Tricot, Paris : Librairie philosophique J. Vrin, 1966, Tome 1, p. 245-247.

7. Ibid., 1012b35-1013a1, p. 245.

8. Ibid., 1013a1-3, p. 245-246.
} 
des choses : d'abord, principe se dit de quelque chose d'intrinsèque à ce qui devient et désigne la première partie construite sur laquelle s'édifie tout le reste, comme les fondations d'une maison, la structure d'un bateau, etc.; puis, principe se dit de quelque chose d'extrinsèque à ce qui devient et correspond à ce qui met en marche une génération quelconque, que ce soit dans les choses naturelles, comme le père et la mère sont principes de l'enfant, dans les choses morales et politiques, comme le chef d'un État est principe d'un changement social, et dans les choses artificielles, la connaissance d'une technique est principe de la fabrication d'une œuvre ou d'un objet. Enfin, un dernier sens de principe désigne «le point de départ de la connaissance d'une chose ... : les prémisses sont les principes des démonstrations ${ }^{9}$ ", ainsi les propositions de départ sont les principes d'une théorie scientifique, comme il a été dit plus haut. À la fin de ce même chapitre, après avoir distingué ces sens du mot principe, Aristote indique ce qu'ils ont en commun : "Le caractère commun de tous les principes, c'est donc d'être la source d'où l'être, ou la génération, ou la connaissance dérive ${ }^{10}$ ». Ce qui revient à dire que lorsqu'on parle de principe, dans quelque domaine que ce soit, c'est à ces termes "premiers", à ces éléments "premiers" d'où tout découle, qu'on se réfère. Et, par définition, c'est cela, le champ d'études du philosophe.

On comprend, dès lors, l'importance de la philosophie, du fait qu'elle cherche à scruter ce qui est tout à fait premier dans l'ordre de la nature et dans celui des diverses activités humaines ${ }^{11}$. Et ce qui est tout à fait premier dans ces divers ordres est le fondement sur lequel s'appuie toute notre compréhension de ce qui nous entoure et le fondement aussi d'où l'homme tire les règles de son comportement. De par sa nature,

9. Ibid., 1013a14-16, p. 247.

10. Ibid., 1013a16-17, p. 247. Je n'aime pas ici le mot source utilisé par le traducteur J. Tricot. Je lui préfère l'expression "premier terme", ce qui est plus proche de l'étymologie du mot principe.

11. Il faut bien noter ici que cette réflexion sur les principes n'est pas l'apanage des seuls philosophes dits "professionnels". Certains savants, certains artistes, certains chefs d'État, etc., s'interrogent sur les principes de leurs activités, et ce faisant, ils font œuvre de philosophe. 
c'est-à-dire en raison de son sujet d'étude, la philosophie doit se mettre à la portée des gens, doit donc être vulgarisée, parce que ses réflexions peuvent être profitables à toutes les activités humaines, à la condition d'être comprises. Ainsi les recherches des savants et des artistes pourraient être vues selon une perspective qui en dévoilerait les valeurs et les limites, qui mettrait en évidence les valeurs morales qu'elles impliquent, et cela autant pour les savants et les artistes eux-mêmes que pour l'ensemble de la population. II en serait ainsi encore pour les grands mouvements politiques et sociaux qui agitent la planète, pour les grands problèmes moraux qui assaillent l'homme, individuellement et collectivement. La philosophie reprendrait ainsi toute sa place, qui est de fournir une lumière - que j'identifierais comme la préoccupation de l'universel - capable d'éclairer l'homme dans les jugements qu'il pose sur tout ce qui le concerne. Cette recherche de l'universel me semble bien être une caractéristique de notre temps, comme le montre assez l'engouement pour les sectes et les gourous de toute espèce. Et si cette recherche de l'universel est tellement généralisée aujourd'hui, c'est en raison des moyens de communication qui nous mettent immédiatement en contact avec tout ce qui se passe; comment réussir à voir un peu clair dans ce bombardement d'informations sans la lumière que peut fournir la philosophie grâce à sa connaissance des principes, et donc de l'universel?

Une première raison, fondamentale, qui fait un devoir aux philosophes de vulgariser leur discipline, tient au sujet particulier sur lequel portent leurs études. II en est une autre qui entraîne la même obligation, même si cette raison n'est pas propre à la philosophie : c'est la façon dont connaît l'intelligence humaine.

Dans son processus de compréhension, l'intelligence est en effet gouvernée par un certain nombre de principes qui exigent d'être respectés si l'on veut véritablement connaître. Remarquons qu'une intelligence, laissée à elle-même, a une tendance naturelle à se conformer à ces principes, comme on peut facilement l'observer dans les recherches des savants. 
C'est dans l'enseignement sous toutes ses formes que parfois ... souvent ... professeurs et étudiants semblent oublier ...

La démarche intellectuelle peut être comparée à la marche naturelle du corps, comme le fait Claude Bernard.

De même que dans la marche naturelle du corps, l'homme ne peut avancer qu'en posant un pied devant l'autre, de même dans la marche naturelle de l'esprit, l'homme ne peut avancer qu'en mettant une idée devant l'autre. Ce qui veut dire, en d'autres termes, qu'il faut toujours un premier point d'appui à l'esprit comme au corps. Le point d'appui du corps, c'est le sol dont le pied a la sensation; le point d'appui de l'esprit, c'est le connu, c'est-à-dire une vérité ou un principe dont l'esprit a conscience. L'homme ne peut rien apprendre qu'en allant du connu à l'inconnu'12.

Ce qu'affirme cet extrait, tout en le manifestant au moyen d'une comparaison, c'est la nécessité d'un point d'appui pour fonder toute démarche intellectuelle. C'est aussi l'identification qui est faite entre ce point d'appui et ce que connaît déjà l'intelligence, et qui lui sert à saisir ce qu'elle ne connaît pas. Illustrons par trois exemples tirés d'une recherche que fit le même Claude Bernard.

On apporta un jour dans mon laboratoire des lapins venant du marché. On les plaça sur une table où ils urinèrent et j'observai par hasard que leur urine était claire et acide. Ce fait me frappa, parce que les lapins ont ordinairement l'urine trouble et alcaline en leur qualité d'herbivores, tandis que les carnivores, ainsi qu'on le sait, ont, au contraire, les urines claires et acides ${ }^{13}$.

Voilà un premier cas où manifestement la connaissance des caractères normaux de l'urine des carnivores et des herbivores permet de constater l'anormalité de l'urine des quelques lapins se trouvant sur une table de son laboratoire. Enlevez cette connaissance et l'observation ne peut être faite, et conséquemment la démarche intellectuelle qui va suivre ne peut pas être effec-

12. Claude Bernard, Introduction à l'étude de la médecine expérimentale, Paris : Garnier-Flammarion, 1966, p. 79.

13. Ibid., p. 216. C'est moi qui souligne. 
tuée et l'idée nouvelle (ou la connaissance nouvelle) résultant de cette démarche ne peut pas non plus être obtenue. Mais avant de parvenir à cette connaissance nouvelle, prend place une démarche proprement dite qui, dans cet exemple, se ramène à deux étapes. D'abord la formulation d'une hypothèse pour tenter d'expliquer cette observation :

\begin{abstract}
Cette observation d'acidité de l'urine chez les lapins me fit venir la pensée que ces animaux devaient être dans la condition alimentaire des carnivores. Je supposai qu'ils n'avaient probablement pas mangé depuis longtemps et qu'ils se trouvaient ainsi transformés par l'abstinence en véritables animaux carnivores vivant de leur propre sang14.
\end{abstract}

L'idée nouvelle qui est mise de l'avant, à savoir l'hypothèse, s'appuie sur une connaissance que possédait obligatoirement son auteur : la connaissance du mode d'alimentation des carnivores et des herbivores qui explique les caractères différents de leurs urines. Encore ici, enlevez ce "connu", et cette hypothèse ne peut être formulée. Enfin, la dernière étape de cette démarche : la vérification de cette hypothèse. "Rien n'était plus facile que de vérifier par l'expérience cette idée préconçue ou cette hypothèse ${ }^{15}$ ». Et de fait, alternativement, il donna à manger à ces lapins et les priva de nourriture, et constata toujours que les caractères de leurs urines changeaient selon le mode d'alimentation; ce qu'il constata aussi chez le cheval, autre herbivore. Le connu, ici, c'est le résultat de chaque expérience, c'est-à-dire la connaissance obtenue par l'observation de ce qui résulte de chaque modification du régime alimentaire. L'ensemble de ces connaissances particulières est donc l'appui sur lequel se fonde l'intelligence pour parvenir à une connaissance générale nouvelle «qui alors n'était pas connue, à savoir qu'à jeun tous les animaux se nourrissent de viande, de sorte que les herbivores ont alors des urines semblables à celles des carnivores ${ }^{16}$ ».

14. Ibid.

15. Ibid. C'est moi qui souligne.

16. Ibid., p. 217. C'est moi qui souligne. 
II est bien évident, dans cet exemple de recherche, que l'intelligence s'accroche continuellement à ce qu'elle connaît déjà pour effectuer sa démarche. Or c'est là un principe, parmi les plus fondamentaux, qui régit tout le processus intellectuel, parce que lié à la nature même de notre intelligence, comme la lumière et la chaleur sont liées à la nature même du soleil. Principe qui entraîne donc la nécessité de "vulgariser» toutes les connaissances, y compris la philosophie, c'est-à-dire de mettre ces connaissances à la portée des auditeurs et des lecteurs, jeunes et vieux, profanes en ces matières, en faisant appel à des notions dont on est certain qu'elles sont connues ou qui sont à la portée de l'observation la plus immédiate. Aligner des idées abstraites, trop universelles, sans rapport avec le connu de l'interlocuteur, c'est semblable à un cosmonaute qui mettrait un pied devant l'autre dans l'espace, ou à un bébé qui gigote, couché dans son berceau : faute de point d'appui, ni l'un ni l'autre ne peut avancer. Ajoutons encore, en nous fondant toujours sur cette comparaison de Claude Bernard, que la certitude de notre connaissance dépend, en partie, de la certitude que nous avons des notions sur lesquelles nous nous appuyons, tout comme l'assurance de la marche corporelle dépend, en partie, de la solidité de l'appui sur lequel se pose le pied. Les conclusions ne peuvent pas être plus certaines que les prémisses qui les fondent.

Un autre principe, tout aussi essentiel à notre propos, fonde toute démarche de l'intelligence : celui qui ressort clairement d'une analyse toute simple de la façon dont nous définissons. En effet, si on me demande de définir une craie et un tableau de classe, le premier mouvement que va faire mon esprit, et cela tout à fait spontanément, c'est de rattacher ces deux réalités bien distinctes à un ensemble plus large, de les confondre d'abord dans une notion plus générale, et je dirai alors que la craie et le tableau sont des objets (ou mieux des instruments); puis je saisirai leur différence en énonçant la fonction propre à chacun de ces instruments : la craie est un instrument qui sert à écrire et le tableau est un instrument sur lequel on écrit. Si maintenant on me demande de poursuivre et de définir la craie et le stylo, je m'apercevrai que craie et stylo sont deux instru- 
ments qui servent à écrire et je devrai donc préciser ce qui différencie les deux en identifiant, par exemple, la matière qui permet l'écriture : le calcaire pour la craie et l'encre pour le stylo. Un dernier pas : définir le stylo et la plume. Il s'agit ici de deux instruments qui servent à écrire avec de l'encre : mon intelligence doit donc rechercher une connaissance plus précise qui permettra de saisir ce qui distingue ces deux objets, et ce pourra être par la description du mécanisme qui permet l'écriture dans chaque cas : une pointe mobile pour le stylo et une pointe fixe pour la plume.

Ces exemples nous montrent assez que l'intelligence, pour définir, commence par confondre l'objet à définir dans une connaissance plus globale - qui est aussi, remarquons-le, quelque chose de connu -, connaissance plus globale qui donne de l'objet à définir une connaissance incomplète : savoir, en effet, que le vibraphone, le xylorimba et le bandonéon sont des instruments de musique ne m'éclaire pas beaucoup sur la nature exacte de chacun, mais fournit une délimitation à mon champ de recherche, c'est un point de départ obligé. Une connaissance plus distincte, plus précise, et partant plus complète est obtenue lorsque je suis parvenu à saisir ce qui distingue le plus complètement possible l'objet à définir de tous ceux qui appartiennent au même groupe.

Ce mouvement de l'intelligence qui va du confus au distinct, de l'incomplet au complet, ne gouverne pas seulement la recherche d'une définition, qui est une recherche relativement simple, mais encore les recherches même les plus longues et les plus complexes effectuées par l'intelligence. En voici un exemple facile à suivre, fourni par Jean-Henri Fabre17. Ayant observé des processionnaires du pin adultes durant toute une matinée, le célèbre entomologiste en reçut un cuisant prurit au visage qu'il attribua aux poils de la chenille, que sa respiration amenait à son visage et que ses mains, essayant de soulager le début des démangeaisons, fixaient davantage à sa peau. Grâce à une expérience directe avec les poils de la procession-

17. Fabre, Jean-Henri, Souvenirs entomologiques, Paris : Librairie Delagrave, 1924, Tome VI, c. XXIII et XXV, p. 407-423, 435-452. 
naire qu'il étendit à la face interne de l'un de ses avant-bras, il constata qu'il en était bien ainsi : le prurit provoqué par l'observation de cette chenille est bel et bien causé par ses poils. Aussitôt une question se forma dans son esprit : comment les poils peuvent-ils causer l'urtication? Après avoir éliminé deux possibilités, il supposa que les poils avaient, à leur surface, un agent d'irritation. Ayant lavé une quantité de poils dans l'éther sulfurique, il fit deux expériences semblables à la précédente : la première avec les poils débarrassés de leur supposé agent d'irritation montra que ceux-ci ne sont pas la cause directe de l'urtication, puisqu'ils ne provoquèrent aucune douleur, alors que la seconde, faite avec le résidu du lavage, filtré et évaporé, prouva le bien-fondé de son hypothèse, car ce résidu provoqua sur son avant-bras des brûlures et des douleurs durant une couple de jours. Cette découverte suscita, à son tour, une nouvelle question : d'où vient cet agent d'irritation ou ce virus? Après avoir éliminé l'existence d'une glande spéciale qui élaborerait ce virus, en raison de la parité de structure interne des chenilles urticantes et des chenilles bénignes, il supposa que ce virus est un déchet de l'organisme qui doit alors se retrouver dans le sang et dans les crottins de la processionnaire du pin. Encore une fois, l'expérience faite à partir de chacune de ces deux matières ne confirme que trop son raisonnement. Mais alors, ce virus, déchet produit par l'organisme de la processionnaire du pin, serait-il exclusif à cette seule chenille? Pourquoi le serait-il? «Ne serait-il pas plutôt propriété commune à toutes les chenilles, aux lisses comme aux vêtues de poils? 18 » Utilisant à nouveau le même procédé, mais cette fois avec le crottin du ver à soie, chenille à la peau lisse et dont le maniement n'occasionne aucun désagrément, il parvient encore au même résultat. II en est de même pour toutes les expériences faites sur d'autres chenilles. Dans ce cas, comment expliquer que certaines chenilles velues soient inoffensives, alors que d'autres sont à craindre? Ici intervient, selon Fabre, le mode de vie des chenilles : certaines, comme la processionnaire du pin, vivent entassées dans des nids où s'accumulent leurs déchets,

18. Ibid., p. 441. 
au contact desquels les poils de la chenille s'enduisent du virus, alors que, au contraire, d'autres, comme la hérissonne, douée d'une pilosité semblable à celle de la processionnaire, sont inoffensives parce qu'elles vivent en solitaires, en-dehors de nids, leurs poils n'entrant donc pas en contact avec leurs crottins.

Arrêtons ici le résumé de cette recherche, qui illustre suffisamment bien comment procède l'intelligence dans l'acquisition de connaissances : elle commence par établir un premier point, simple, approximatif (savoir que l'urtication est causée par les poils de la processionnaire n'est manifestement pas ce qui fournit l'explication complète de ce phénomène), à partir duquel elle parviendra, peu à peu, à une explication de plus en plus complète et précise, tenant compte de la complexité et de la totalité du phénomène à comprendre. Est-il nécessaire d'ajouter que ce processus de l'intelligence, qui va d'une connaissance confuse à une connaissance de plus en plus distincte, ne se vérifie pas seulement dans le domaine de la science, mais dans l'acquisition de toute connaissance, parce que c'est là un principe qui n'est pas lié à la matière étudiée, mais au fonctionnement même de cette faculté? Voilà donc une troisième raison qui entraîne la nécessité de vulgariser la philosophie. Vouloir présenter un principe, à des profanes, en faisant intervenir toutes les subtilités auxquelles une connaissance plus précise conduit inévitablement, c'est se condamner à n'être pas compris. Le philosophe - ou soyons plus humbles : le professeur de philosophie - doit rechercher, et posséder cette connaissance la plus précise possible des principes, pour pouvoir identifier ce qui doit, de toute nécessité, être expliqué à l'occasion de premières approches, mais il ne doit pas viser, répétons-le, à transmettre cette connaissance précise au début. Pour jouer efficacement son rôle dans l'ensemble de la société, le philosophe doit donc impérativement s'en tenir à engendrer une connaissance certaine, évidente, même si elle est incomplète et ne comporte pas toutes les distinctions qu'exigerait une connaissance distincte des principes en jeu dans les débats sociaux, politiques et moraux qui animent nos sociétés. Un travail semblable doit aussi être fait concernant les principes qui sont à la base des diverses sciences, et concernant les 
principes qui règlent la vie morale individuelle. Ce n'est que lorsque cette première étape sera assurée qu'on pourra passer à une autre. Ce qui ne semble pas être encore le cas, si on porte attention au type de débats qui ont cours dans nos sociétés, où les fantaisies intellectuelles, les options politiques, les opinions personnelles ou les impulsions du moment tiennent trop souvent lieu d'arguments.

Trois raisons essentiellement commandent aux philosophes de vulgariser leur discipline. La première, rappelons-le, découle du sujet sur lequel porte la réflexion philosophique : parce que les principes, étudiés par les philosophes, sont la base tout à fait fondamentale qui éclaire les diverses activités humaines, qui permet de justifier les choix que l'homme fait dans ses activités, et qui permet de juger ces activités elles-mêmes et la valeur des conséquences qui en découlent, ces principes doivent donc impérativement être accessibles à tous les êtres humains. Les philosophes doivent donc trouver des moyens pour mettre en lumière, et de façon compréhensible par tous, les principes en cause dans les divers débats politiques, scientifiques, sociaux, moraux, etc. Et puisque ces principes intéressent au plus haut point tous les hommes, le travail du philosophe sera efficace dans la mesure où il sera vulgarisé, c'est-à-dire dans la mesure où il respectera ces deux grands principes qui ont été précédemment illustrés et qui régissent la démarche intellectuelle de tout homme.

En corollaire, on doit noter que ces trois raisons qui obligent le philosophe à présenter ses études de façon accessible, permettent aussi de définir la vulgarisation philosophique et de juger certaines tentatives qui ont été faites ou qui ont été présentées comme telles. Par exemple, le roman de Jostein Gaarder, Le Monde de Sophie19, n'est pas une vulgarisation de cette sorte, parce que ce qui est abordé dans ce livre, c'est la progression des questions philosophiques et non la présentation de principes. C'est une belle vulgarisation, même si elle est un peu simpliste, de l'histoire de la philosophie, mais 
certainement pas de la réflexion philosophique comme telle sur son sujet. Autre exemple : Le fondement de la morale d'André Léonard 20 . Livre très intéressant en maints endroits, et qui traite bien de principes en morale, mais d'une manière disproportionnée pour le lecteur qui n'a pas de connaissances un peu poussées des divers systèmes philosophiques : continuellement, en effet, l'auteur fait appel à des notions développées par des philosophes pour manifester ses propres thèses. Bien sûr, le philosophe doit s'adresser à tous les "publics", mais il ne s'agit certainement plus de vulgarisation quand on présuppose une connaissance assez précise de l'histoire de la philosophie. Certaines autres tentatives me semblent tenir davantage des salons littéraires du XIXe siècle, alors que d'autres relèvent carrément du spectacle...

En somme, ce que montrent ces quelques remarques sur la vulgarisation de la philosophie, c'est la nécessité d'engendrer des racines, c'est-à-dire quelques connaissances simples mais bien ancrées dans l'esprit, qui deviendront des instruments de jugement et des règles de direction dans divers aspects de la vie. Dans un plan plus large, c'est un travail qui consiste à identifier et à exposer simplement les principes en cause dans telle situation ou tel problème qui intéresse une société, car il doit bien y avoir autre chose que la seule dimension économique à la base, par exemple, de l'indépendance du Québec, de la réforme de l'éducation, de celle des soins de santé, etc. II faut bien se convaincre que ce n'est pas rabaisser la philosophie que de faire œuvre de vulgarisation, mais plutôt renouer avec un de ses rôles clairement perçu à son origine même, spécialement par Socrate qui compare son rôle auprès de ses concitoyens à celui d'un taon vis-à-vis "un grand cheval de bonne race ${ }^{21 » \text {. }}$

\section{Lucien Bérubé Collège de Sainte-Foy}

20. André Léonard, Le fondement de la morale, Paris : Les Éditions du Cerf, 1991, 383 pages.

21. Platon, Apologie de Socrate, 30e - 31b, traduction de Frédérick Têtu, Québec : Collection Résurgences, 1995, p. 39. 